\title{
Investigation on Wearable Antenna under Different Bending Conditions for Wireless Body Area Network (WBAN) Applications
}

\author{
Ismahayati Adam (D), ${ }^{1}$ Muhammad Ramlee Kamarudin $\mathbb{D}^{2},{ }^{2}$ Ali H. Rambe, ${ }^{3}$ \\ Norshakila Haris, ${ }^{4}$ Hasliza A. Rahim, ${ }^{1}$ Wan Zuki A. Wan Muhamad ${ }^{5},{ }^{5}$ Arif M. Ismail, ${ }^{1}$ \\ Muzammil Jusoh, ${ }^{1}$ and Mohd Najib M. Yasin $\mathbb{D D}^{1}$ \\ ${ }^{1}$ Advanced Communication Engineering Research Centre, Faculty of Electronic Engineering Technology, \\ Universiti Malaysia Perlis, Arau 02600, Perlis, Malaysia \\ ${ }^{2}$ Faculty of Electrical and Electronic Engineering, Universiti Tun Hussein Onn Malaysia, 86400 Parit Raja, \\ Johor, Malaysia \\ ${ }^{3}$ Department of Electrical Engineering, Universitas Sumatera Utara, Medan, Indonesia \\ ${ }^{4}$ Universiti Kuala Lumpur Malaysian Institute of Marine Engineering Technology, Sitiawan 32200, Perak, Malaysia \\ ${ }^{5}$ Faculty of Applied and Human Science, Universiti Malaysia Perlis, Arau 02600, Perlis, Malaysia
}

Correspondence should be addressed to Mohd Najib M. Yasin; najibyasin@unimap.edu.my

Received 28 January 2021; Revised 28 April 2021; Accepted 28 May 2021; Published 10 June 2021

Academic Editor: Mauro Parise

Copyright (C) 2021 Ismahayati Adam et al. This is an open access article distributed under the Creative Commons Attribution License, which permits unrestricted use, distribution, and reproduction in any medium, provided the original work is properly cited.

\begin{abstract}
This paper analysed the effects of bending on the performance of a textile antenna wherein the antenna under test was made of felt substrate for both industrial, scientific, and medical (ISM) band and WBAN applications at 2.45 GHz. Moreover, the conductive material was used for the patch, and the ground plane used a $0.17 \mathrm{~mm}$ Shieldit textile. Meanwhile, the antenna structure was in the form of rectangular, with a line patch in between elements to abate the mutual coupling effect. The measured operating frequency range of the antenna spanned from $2.33 \mathrm{GHz}$ to $2.5 \mathrm{GHz}$ with a gain of $4.7 \mathrm{dBi}$ at $2.45 \mathrm{GHz}$. In this paper, the antenna robustness was examined by bending the structure on different radii and degrees along both $X$ - and $Y$ axis. Next, the effects on return loss, bandwidth, isolation, and radiation characteristics were analysed. This paper also discovered that the antenna's performance remained acceptable as it was deformed, and the measured results agreed well with the simulation.
\end{abstract}

\section{Introduction}

The concept of wearable antenna design was originated from the idea of having wearable computing systems as part of clothing that provides the wearer with hassle-free continuous data transfer. Extensive research studies have investigated the realisation of wireless body area networks (WBANs), specifically on the textile antenna design in medical and nonmedical applications. On top of that, the emergence of $5 \mathrm{G}$ technology creates multiple prospects of WBAN applications, including child protection, tracking, military, real-time health monitoring, biosensors, tourism, and security [1-4].
Furthermore, the incorporation of antennas and flexible electronics into clothes as a body-worn device eliminates the need for carrying a device, thus providing comfortability to the wearer [1]. Besides, in terms of sustainability of the wearable device, it should be lightweight, offer great flexibility, inexpensive, and robust. Considering these characteristics, this paper proposed a simple structure of rectangular patch multiple-input multiple-output (MIMO) antenna [5-9]. It should be noted that MIMO systems are extensively used in wireless communication to optimise the system with greater signal receptions and enlarge channel capacity [10]. 
For a flexible antenna, bending is a vital parameter as it could experience bending and crumpling during practical application. Additionally, a wearable dual-band antenna with an artificial magnetic conductor (AMC) structure on the back is proposed to alleviate body coupling [3]. It could be observed that the antenna reflection coefficient was conserved better during $Y$-axis bending compared with $X$ axis bending. Works in [4] showed an interesting finding on the bending effect of the antenna performance with different dielectric values for substrate, where the results showed that the substrate with dielectric constant close to air $\left(\varepsilon_{r}=1\right)$ has stable performance. Research on the impact of different substrate thicknesses (from $2 \mathrm{~mm}$ to $10 \mathrm{~mm}$ ) towards bending conditions was explored in [11]. In addition, bending the antenna resulted in moving $S_{11}$ to a higher frequency, where a thickness of $6 \mathrm{~mm}$ was less affected by the changes when the antenna was deformed. The antenna characteristic is preserved during $64 \mathrm{~mm}$ radius $X$-axis bending with the proposed metamaterial-inspired isolator that combines the defected ground structures (DGSs) and modified split-ring resonators (SRRs) [12]. A study on cylindrically bending hollow and solid effects on fabric-based antenna showed that gain and radiation patterns are almost identical for solid and hollow cylinders under bending, with slightly decreased bandwidth on solid cylinders [13].

This paper presents a bending assessment on different radii and degrees to gauge the impact on the performances of the antenna in [14]. Bending in both $X$ - and $Y$-axis planes was performed by placing the antenna on cylinders with different radii $(25,45,55$, and $65 \mathrm{~mm})$, and their effects were investigated. Simulations against the human phantom model and folded bending were also analysed on the antenna. To the best of the author's knowledge, there is no research available on the folded bending for wearable antenna design.

\section{Materials and Methods}

The antenna bending is characterised by the bending radius, $R$, as illustrated in Figure 1. Two types of bending were explored in this paper, the circular radius bending and degree bending in two principal planes, namely, $X$-axis and $Y$-axis bending. Figure 1(a) shows the antenna in flat; Figure 1 (b) shows the antenna in circular bent in $X$-axis; and Figure 1(c) shows the antenna in degree bending in $Y$-axis. In this paper, the antenna is designed using computer simulation technology (CST) software, where $R$ varied from $65 \mathrm{~mm}$ to $25 \mathrm{~mm}$ (worst case).

The proposed antenna structure is initiated from a rectangular patch, with the patch dimension of $56.6 \times 47 \mathrm{~mm}^{2}$. The antenna is modelled using felt substrate with a dielectric constant of 1.44 and thickness of $3 \mathrm{~mm}$, where Shieldit super with $0.17 \mathrm{~mm}$ thickness is used as the radiating part. Resonating at a frequency of $2.4 \mathrm{GHz}$, the antenna is improved by converting into $2 \times 1 \mathrm{MIMO}$ with the total dimension of $140 \times 70 \mathrm{~mm}^{2}$. A small gap of $0.1 \lambda$ between patch elements was achieved with the introduction of a line patch of $4 \mathrm{~mm}$ width that assists on mutual coupling suppression [15]. A detailed description of the proposed antenna is described in [14].

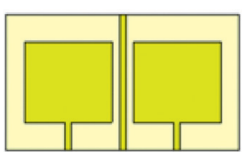

(a)

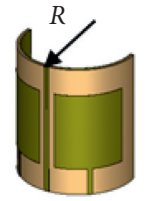

(b)

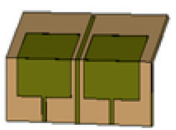

(c) $R=$ radius bending
FIgURE 1: The illustration of the antenna in different conditions: (a) flat; (b) $X$-axis circular bending; (c) $Y$-axis bending.

\section{Results and Discussion}

3.1. Circular Bending. The analysis starts with a circular bending where the antenna was bent in the $Y$-axis and $X$-axis with the radii of 65,55 , and $45 \mathrm{~mm}$. Figure 2 shows the scattering parameter results when the antenna was bent in a different radius. Compared with the result of the antenna in a flat state, the $S_{11}$ curve shifted to the right as the radius decreases from $65 \mathrm{~mm}$ to $25 \mathrm{~mm}$ for $Y$-axis bending as in Figure 2(a). On the other hand, the isolation of the antenna (shown by the $S_{21}$ result) was improved for a smaller radius of bending.

Meanwhile, for $X$-axis circular bending, the resonance frequency shifted to the right as the radius decreased from $65 \mathrm{~mm}$ to $25 \mathrm{~mm}$, as shown in Figure 2(b). On the positive side, the isolation of the antenna (shown by the $S_{21}$ result) was improved by a smaller radius of bending due to the separation between radiating elements that get bigger as the degree bend increases.

Figure 3 shows the variation of off-body radiation pattern concerning the bending radius in E-plane and $\mathrm{H}$-plane. In the $Y$-axis bending, the pattern is almost similar to the direction of radiation. However, the back lobe gets bigger as the bending degree increases (decrease in radius). On another note, the radiation pattern for $X$ axis bending tuned the angle of the main beam for about $30^{\circ}$ in E-plane, while the pattern exhibits back lobe in $\mathrm{H}$-plane when the antenna is bent into a different degree. Regarding gain for both axis bending, the gain decreases as the bending degree increases from radius $65 \mathrm{~mm}$ to $25 \mathrm{~mm}$.

Table 1 compares the gain for different circular bending radii for both $X$-axis and $Y$-axis. Decreasing the circular radius bending will degrade the gain of the antenna. There are noticeable $16 \%$ and $12 \%$ gain reduction in the $X$-axis and $Y$-axis bending from flat to bending of $25 \mathrm{~mm}$ radius, respectively. To validate the MIMO performance, the envelope correlation coefficient (ECC) and diversity gain (DG) were monitored, as shown in Figure 4. ECC implies the degree of independence of the radiation patterns of two antennas [16], while the DG is the signalto-noise ratio. Both parameters were related and calculated from $S$-parameter of radiation far field pattern results. Low ECC will result in high DG, where ECC $<0.5$ is indicated as a good MIMO antenna [15]. In this work, calculated from $S$-parameter values from $2.4 \mathrm{GHz}$ to $2.5 \mathrm{GHz}$ range based on the proposed antenna, the values of ECC were below 0.01 with diversity gain close to 10 in all bending conditions. 


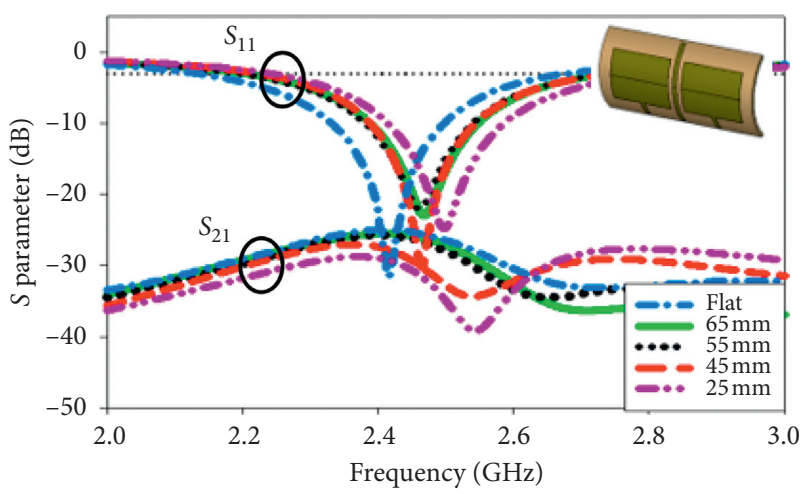

(a)

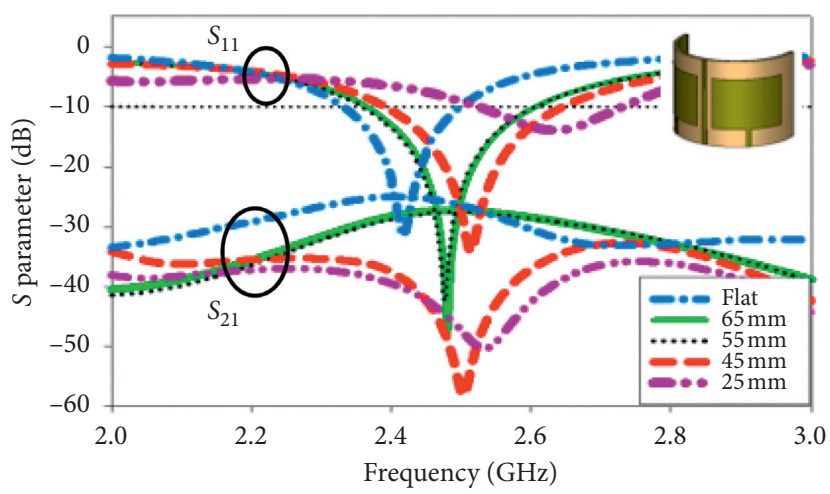

(b)

FIGURE 2: $S$-parameter result with a variation of bending radius in (a) $Y$-axis and (b) $X$-axis.

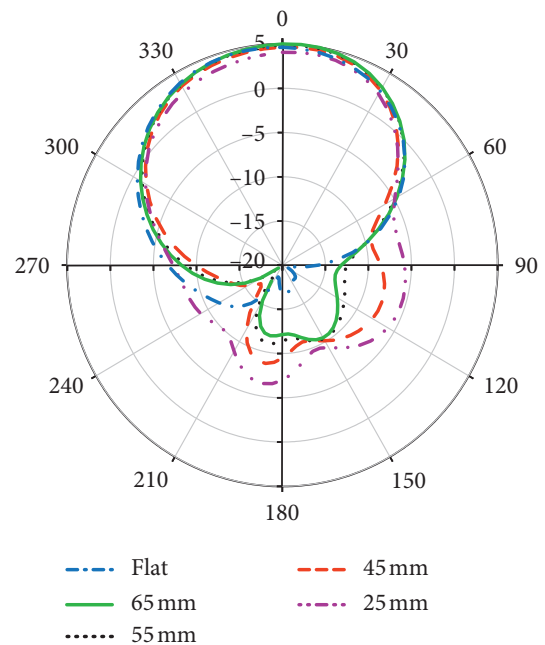

(a)
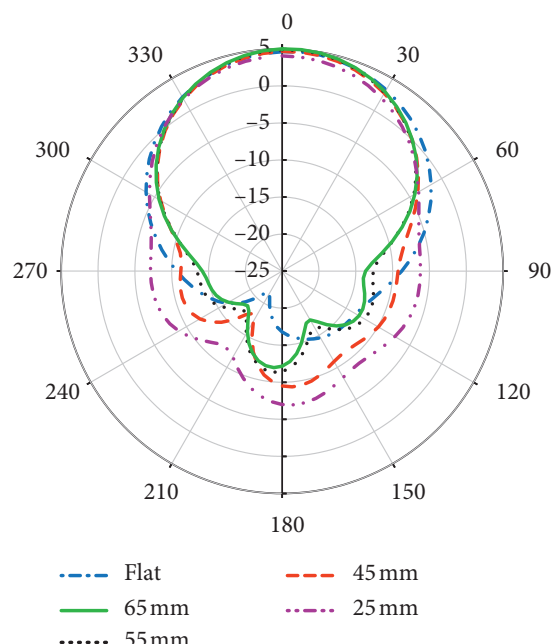

--- $45 \mathrm{~mm}$

.... $25 \mathrm{~mm}$

(b)

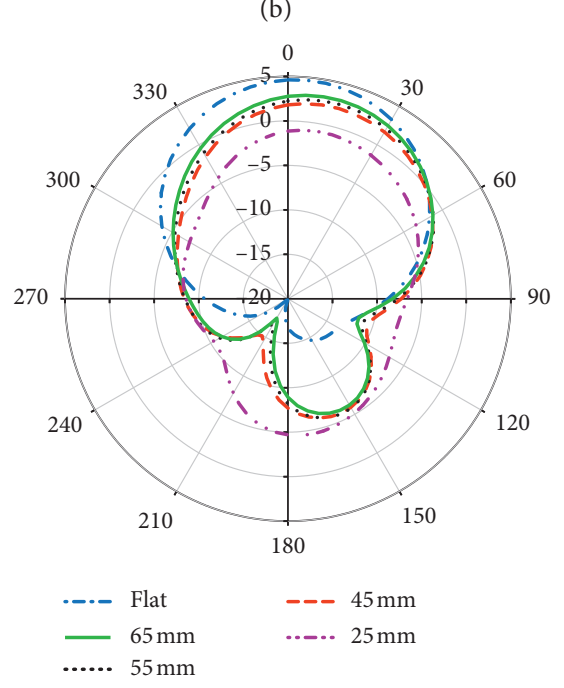

(d)

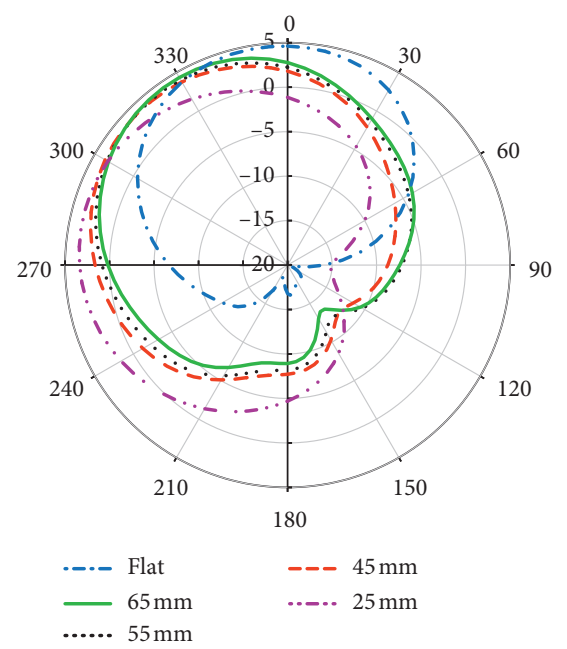

(c) 
TABLe 1: Gain for different circular bending radii.

\begin{tabular}{|c|c|c|c|c|c|c|c|c|c|}
\hline \multirow{3}{*}{ Freq $(\mathrm{GHz})$} & \multicolumn{9}{|c|}{ Radius } \\
\hline & \multirow{2}{*}{ Flat $(\mathrm{dBi})$} & \multicolumn{4}{|c|}{$X$-axis bend gain $(\mathrm{dBi})$} & \multicolumn{4}{|c|}{$Y$-axis bend gain $(\mathrm{dBi})$} \\
\hline & & $25 \mathrm{~mm}$ & $45 \mathrm{~mm}$ & $55 \mathrm{~mm}$ & $65 \mathrm{~mm}$ & $25 \mathrm{~mm}$ & $45 \mathrm{~mm}$ & $55 \mathrm{~mm}$ & $65 \mathrm{~mm}$ \\
\hline 2.4 & 4.7 & 3.93 & 4.38 & 4.51 & 4.72 & 4.13 & 4.72 & 4.95 & 5.05 \\
\hline 2.45 & 4.72 & 3.81 & 4.23 & 4.38 & 4.62 & 4.07 & 4.6 & 4.83 & 4.94 \\
\hline 2.5 & 4.73 & 3.65 & 4.05 & 4.2 & 4.47 & 4.01 & 4.41 & 4.66 & 4.78 \\
\hline
\end{tabular}

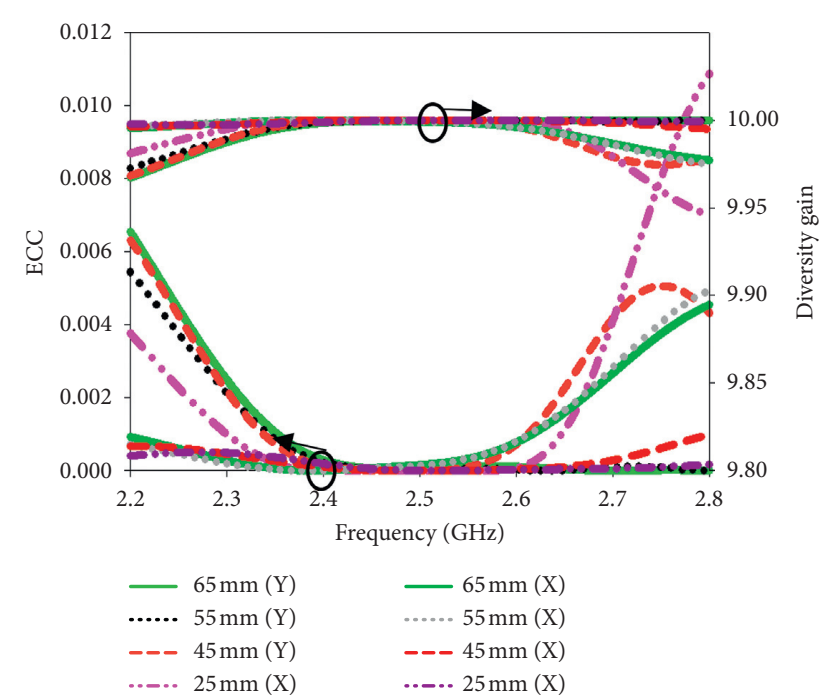

FIgURE 4: ECC and diversity gain for the various radii of circular bend.

$Y$-axis as illustrated in Figure 5. Notably, the model has different dielectric properties and conductivity based on the type of human tissues and frequency. Table 2 shows the values at $2.4 \mathrm{GHz}$.

The simulated result is presented in Figure 5(a), where bending of the antenna to the human model has shifted the resonance to a higher frequency, which also degrades the matching of the antenna. Furthermore, the antenna resonates at $2.49 \mathrm{GHz}(-20 \mathrm{~dB})$ when the structure is bent in $Y$ axis to the human model, while for $X$-axis bending, $S_{11}$ was shifted to $2.58 \mathrm{GHz}$ with the value of $-13 \mathrm{~dB}$. As for mutual coupling, $S_{21}$, the $Y$-axis bending improved the value from -28 to $-36 \mathrm{~dB}$.

On the other hand, the angle of the radiation pattern was shifted about $30^{\circ}$ for $X$-axis, while it maintained the same direction during the $Y$-axis bending. These results are proved in Figure 6 . It should be highlighted that the specific absorption rate (SAR) assessment was used to determine the limit of radiation exposure to the human body. The effect of SAR can be reduced by a large ground plane. Apparently, the proposed antenna is less affected by the human body proximity compared with the omnidirectional antenna as the full ground plane shields the radiating pattern to the human body, which also contributed to low specific absorption rate (SAR) values [17-19]. Hence, in this work, no SAR simulation was conducted.
3.3. Folded Bending. The bending characteristic of the antenna was also analysed in terms of folding degree, ranging from $25^{\circ}$ to $90^{\circ}$. In the $Y$-axis folding degree, the reflection coefficient was shifted to the higher frequency as the bending degree increases from $0^{\circ}$ (flat) to $90^{\circ}$. The result is shown in Figure 7 , where the resonant frequency shifted from $2.42 \mathrm{GHz}$ to $2.512 \mathrm{GHz}$ when the antenna is bent to $90^{\circ}$, whereas the isolation is shiftless at $-26 \mathrm{~dB}$.

In contrast, the result for $X$-axis bending is almost consistent from $25^{\circ}$ to $90^{\circ}$ bent regarding the impedance bandwidth with less than $1 \%$ different in $S_{11}$ result, with better matching as the bending degree increased. A similar trend could be seen in the $S_{21}$ result, where the isolation improved from $-26 \mathrm{~dB}$ to $-38 \mathrm{~dB}$. Also, the radiation pattern is another parameter used to evaluate the antenna performances, and the different degree bending patterns are depicted in Figure 8. There are prominent changes in the $Y$ axis bending antenna pattern, where the values decline with a shifted angle of about $30^{\circ}$ in $\mathrm{H}$-plane. Conversely, the front lobe pattern maintained in the $X$-axis degree bending was slightly bigger in the back lobe as the degree bending increased. Table 3 tabulates the gain result for different degrees in $X$ - and $Y$-axes. For most of the results, the gain decreases as the bending degree increases. Nevertheless, for $Y$-axis bending of $45^{\circ}$, there is a $5 \%$ increment in the gain. Another important observation is that the MIMO performance matrices are maintained during the folded bend of the antenna (see Figure 9), where the antenna exhibits lower ECC and good diversity gain.

3.4. Measurement Result. For the purpose of verifying the antenna characteristic, the antenna was fabricated, and its performance was measured. The antenna was fabricated by manually cutting the textile based on the size as in the simulation, as displayed in Figure 10. The fabricated antenna showed good conformity between simulated and measured results. However, the measured $S_{11}$ shifted to the right of about $2.8 \%$, and there was a $1 \mathrm{~dB}$ increment of $S_{21}$, as shown in Figure 11(a). The shifted operating frequency is due to the fabrication in accuracy. On another note, the measured radiation pattern agreed well with the simulated result, as in Figure 11(b). Figure 11(b) shows the measured radiation pattern which agreed well with the simulated result.

For the bending measurement setup, a PVC pipe with a diameter of $90 \mathrm{~mm}$ and a plastic film was folded into a circular structure of $55 \mathrm{~mm}$ radius. This setup was done to hold the antenna into the circular structure. The 


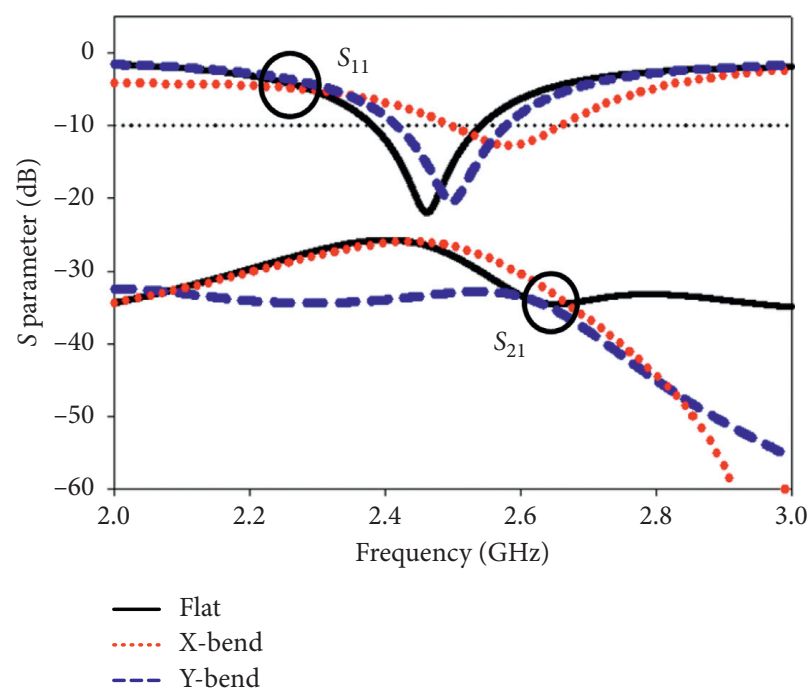

(a)
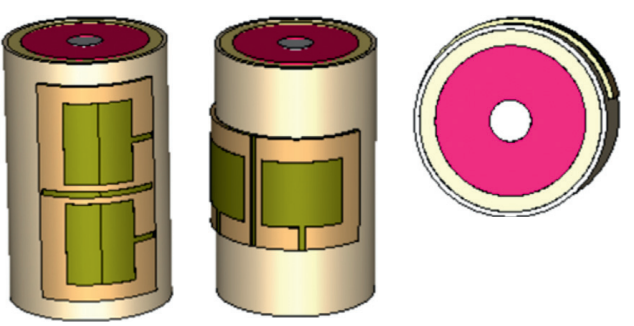

(b)

(c)

(d)

Figure 5: Antenna bent over the human phantom model: (a) return loss and mutual coupling results for on-body simulation; the antenna is bent over; (b) $Y$-axis and (c) $X$-axis; and (d) bottom view of the human model.

Table 2: Properties of human tissues at $2.4 \mathrm{GHz}$.

\begin{tabular}{lcc}
\hline Tissue & $\varepsilon_{r}$ & Conductivity $($ S/m) \\
\hline Skin & 38.06 & 1.4407 \\
Fats & 5.28 & 0.10235 \\
Muscle & 52.79 & 1.705 \\
Bone & 11.41 & 0.38459 \\
\hline
\end{tabular}

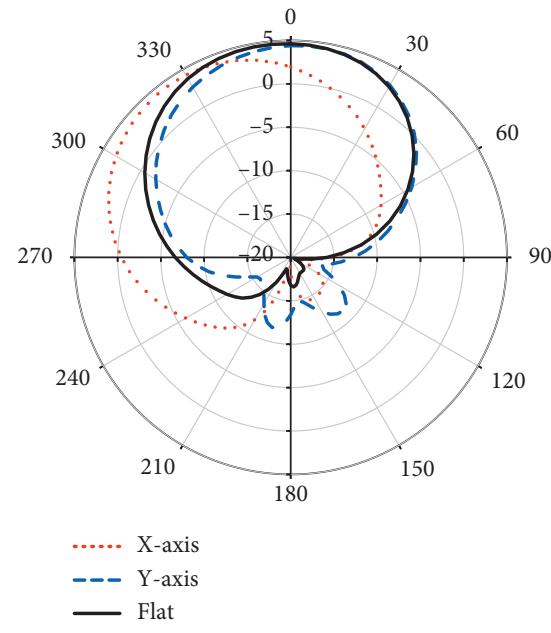

(a)

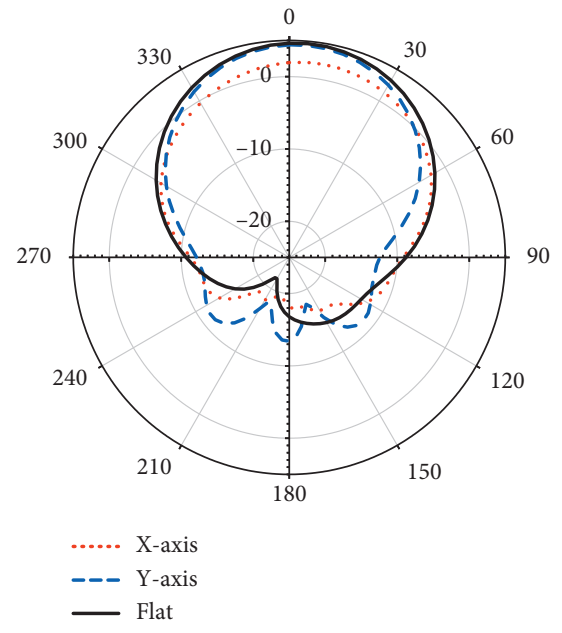

(b)

FIgURE 6: Simulated radiation pattern of phantom in (a) E-plane and (b) H-plane.

measurement setup is depicted in Figure 12, where the antenna is measured using a 2-port measurement of Agilent Vector Network Analyser (VNA). From the observation on the graph plotted in Figure 13, the $S$-parameter results exhibit similar patterns for simulation and measurement but with a small divergence in the values. Meanwhile, for the $45 \mathrm{~mm}$ circular bend, the measured resonance frequency is unchanged irrespective of the bend axis; however, it reduced the matching and impedance bandwidth compared with the simulation. In the $55 \mathrm{~mm}$ circular bend, the centre frequency of both measured $S_{11}$ in $X$ - and $Y$-axis was shifted to $2.49 \mathrm{GHz}$, with $4 \%$ impedance bandwidth. Contrastingly, the $S_{21}$ values were improved to -31 and $-29 \mathrm{~dB}$ compared with the $-26 \mathrm{~dB}$ in simulation for $X$ - and $Y$-axis, respectively. 


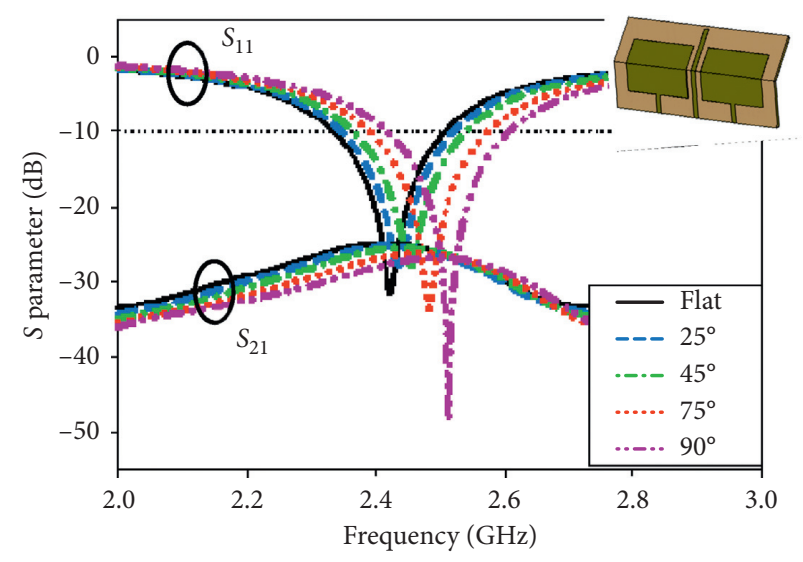

(a)

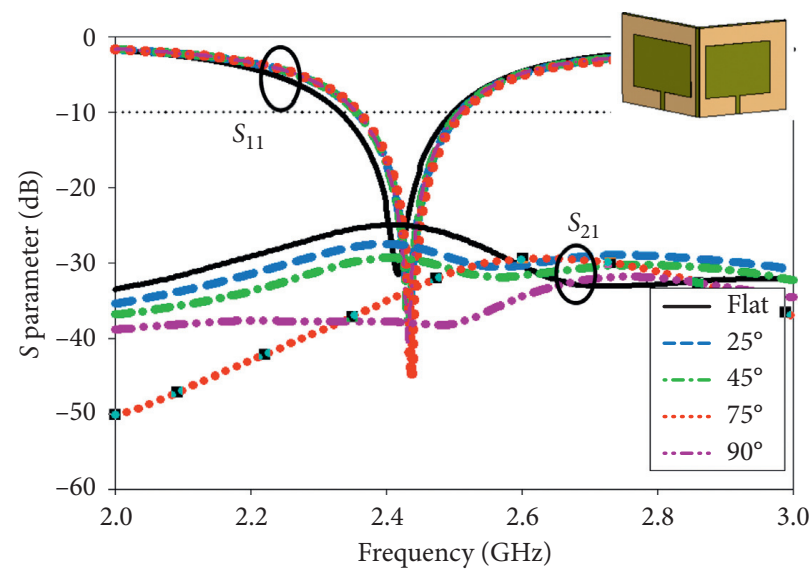

(b)

Figure 7: Reflection coefficient and isolation result with variation in degree bending: (a) $Y$-axis and (b) $X$-axis.

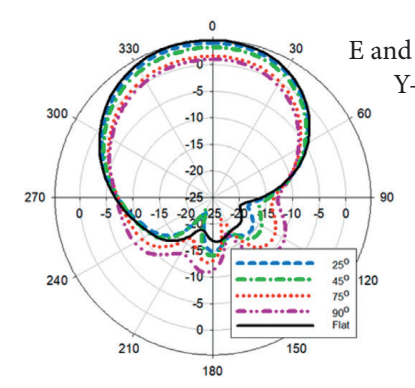

(a)

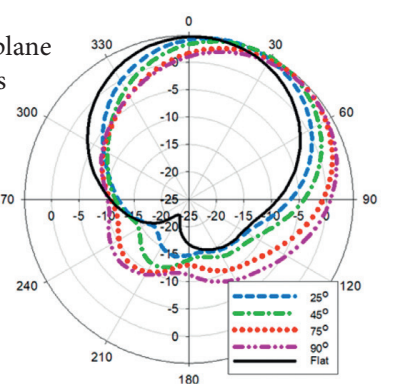

(b)

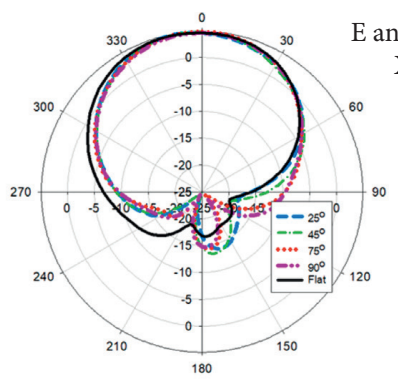

(c)

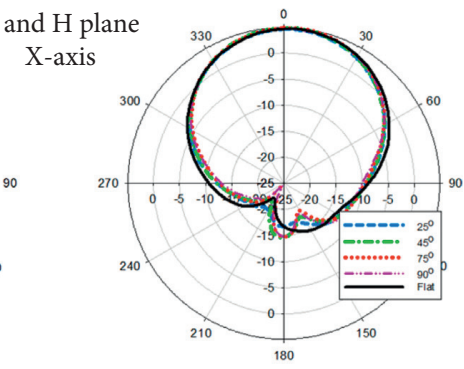

(d)

Figure 8: Radiation pattern of E- and H-plane for different degree bending in $Y$-axis and $X$-axis: (a) E-plane $Y$-axis; (b) H-plane $Y$-axis; (c) E-plane $X$-axis; (d) H-plane $X$-axis.

TABLE 3: Gain comparison for different degree bending in $X$-axis and $Y$-axis.

\begin{tabular}{lcccccccccc}
\hline & \multicolumn{10}{c}{ Degree } \\
Freq $(\mathrm{GHz})$ & \multicolumn{9}{c}{$X$-bend gain $(\mathrm{dBi})$} & \multicolumn{3}{c}{$Y$-bend gain $(\mathrm{dBi})$} \\
& Flat & $25^{\circ}$ & $45^{\circ}$ & $75^{\circ}$ & $90^{\circ}$ & $25^{\circ}$ & $45^{\circ}$ & $75^{\circ}$ & $90^{\circ}$ \\
\hline 2.4 & 4.70 & 4.75 & 4.86 & 4.87 & 4.64 & 4.82 & 4.89 & 4.59 & 4.37 \\
2.45 & 4.72 & 4.60 & 4.70 & 4.66 & 4.41 & 4.87 & 4.95 & 4.64 & 4.42 \\
2.5 & 4.73 & 4.30 & 4.38 & 4.33 & 4.02 & 4.91 & 5.01 & 4.70 & 4.47 \\
\hline
\end{tabular}

For on-body measurement, the antenna was attached to the human upper arm, and the antenna ports were connected to Port 1 and Port 2 of VNA for $S$-parameter evaluation. The results shown in Figure 14 indicate that the measured $S_{11}$ was shifted to the left if compared with the simulation result for $X$ - and $Y$-axes bending. The variance in the results could be a result of simulation, where the antenna was bent directly to the human skin while during the measurement, the antenna and the human skin were separated with a thin fabric layer. In view of isolation, a similar trend was demonstrated for simulation and measurement results with the lowest $S_{21}$ value given by a simulation of $X$-bend.

The performance of the measured antenna is summarized and compared with the other related works in Table 4.

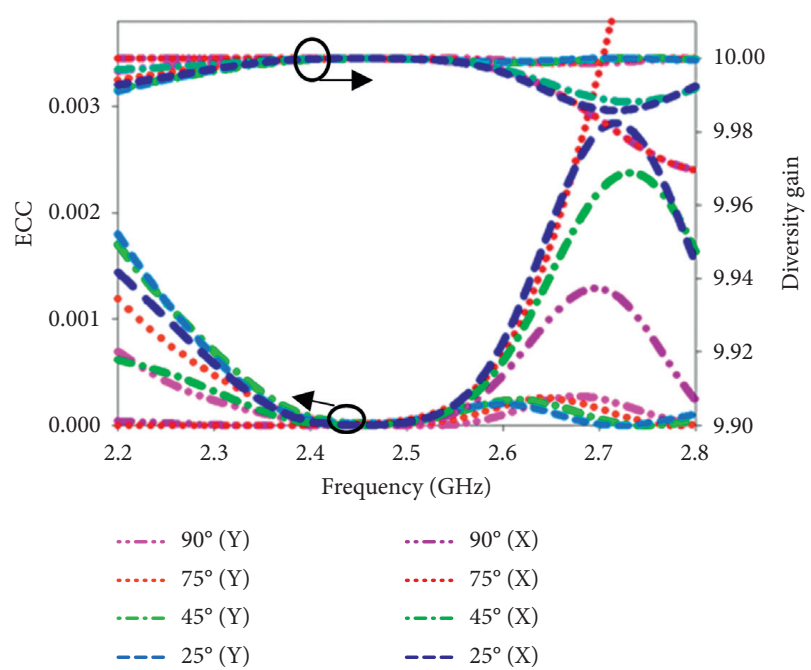

FIgURE 9: ECC and diversity gain for various degrees of folded bend.

The data are based on circular bending experiments, except for [20], where there was no analysis on the bending case. Also, there was no significant deviation on the finding during the on-body experiment, as reported in 


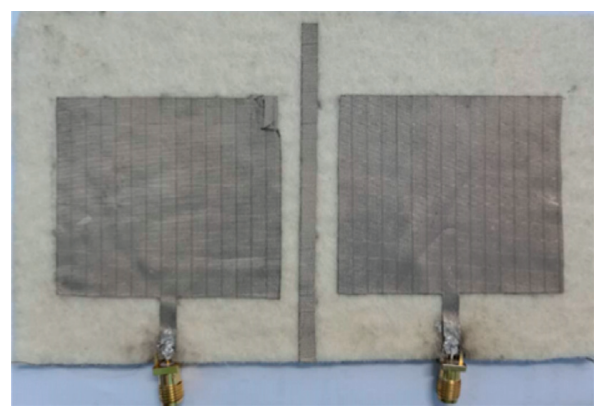

(a)

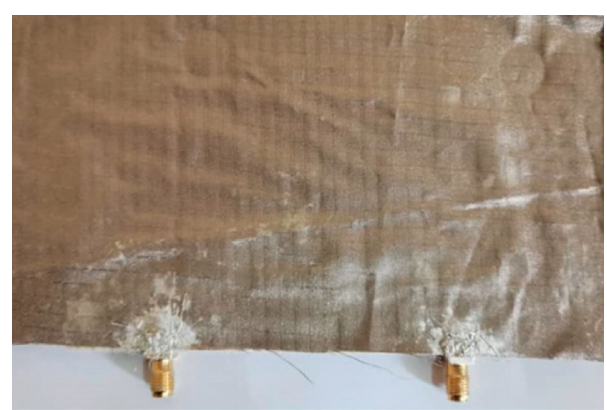

(b)

Figure 10: The fabricated antenna (a) front view and (b) back view.

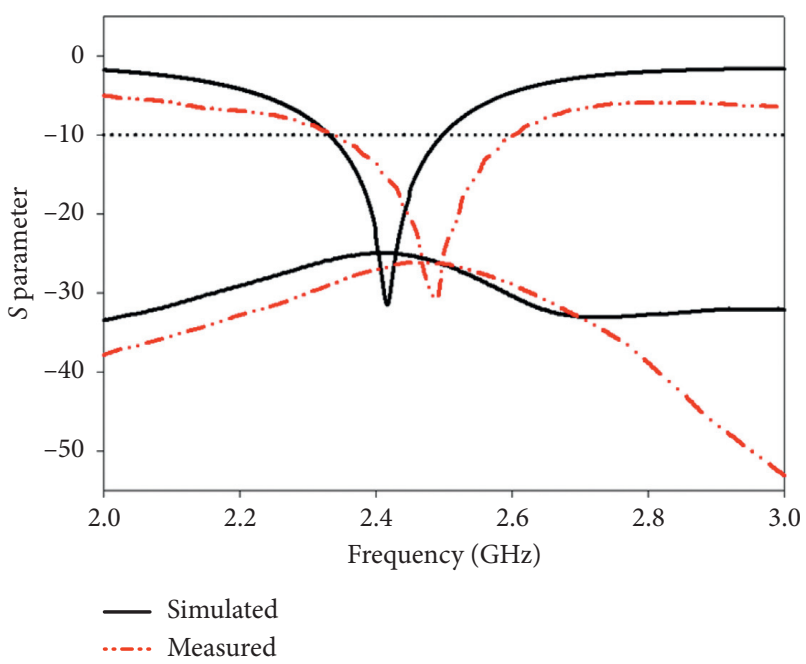

(a)

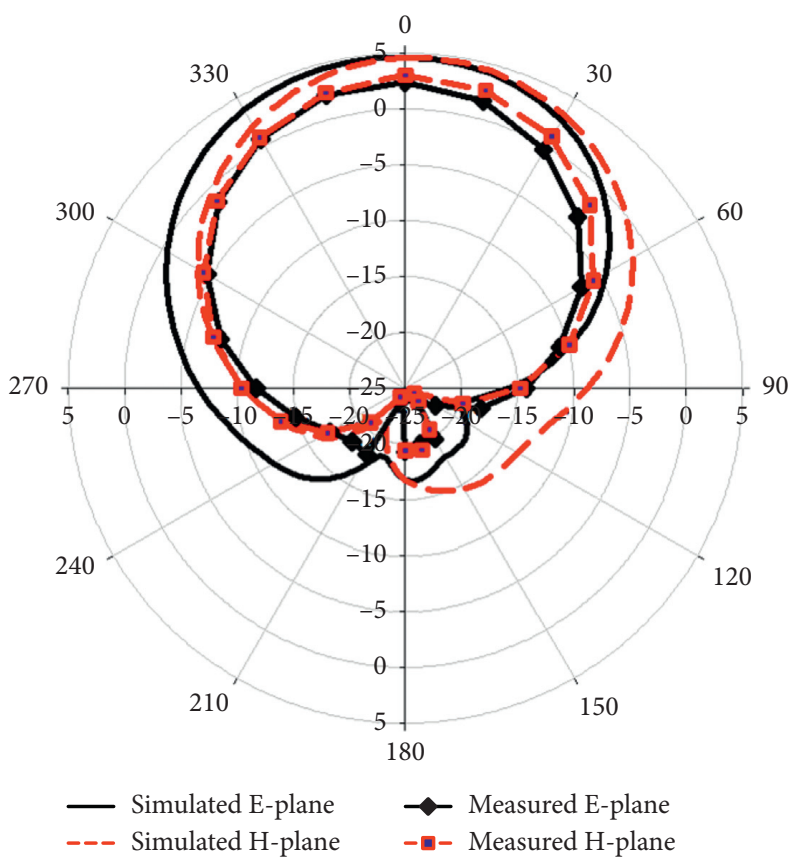

(b)

FIGURE 11: Result comparison of simulation and measurement: (a) $S$-parameter and (b) radiation pattern.

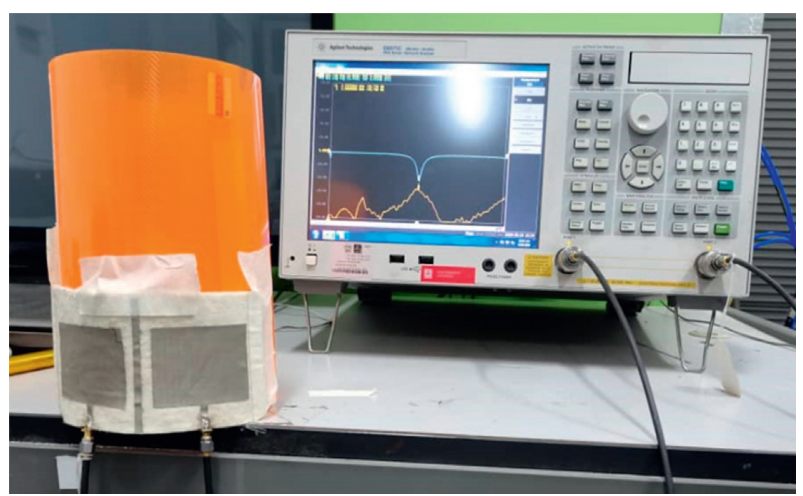

(a)

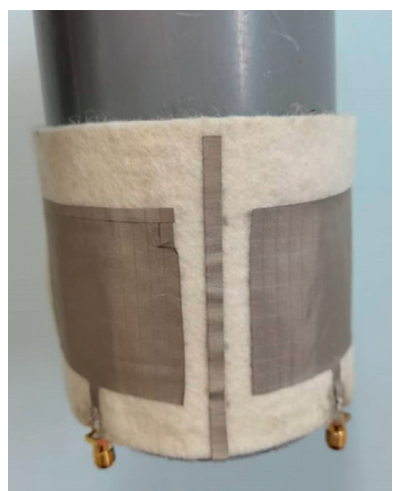

(b)

Figure 12: Bending measurement setup: (a) $55 \mathrm{~mm}$ circular bent in $X$-axis and (b) $45 \mathrm{~mm}$ circular holder. 


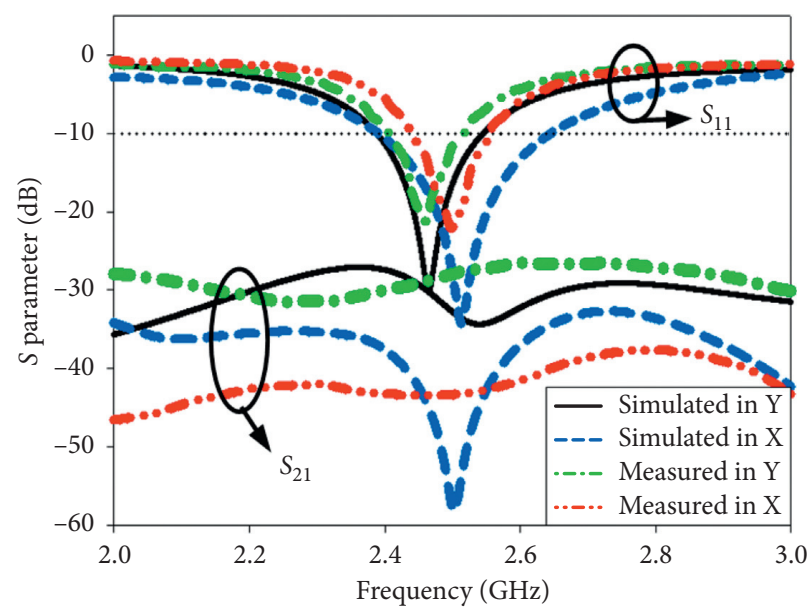

(a)

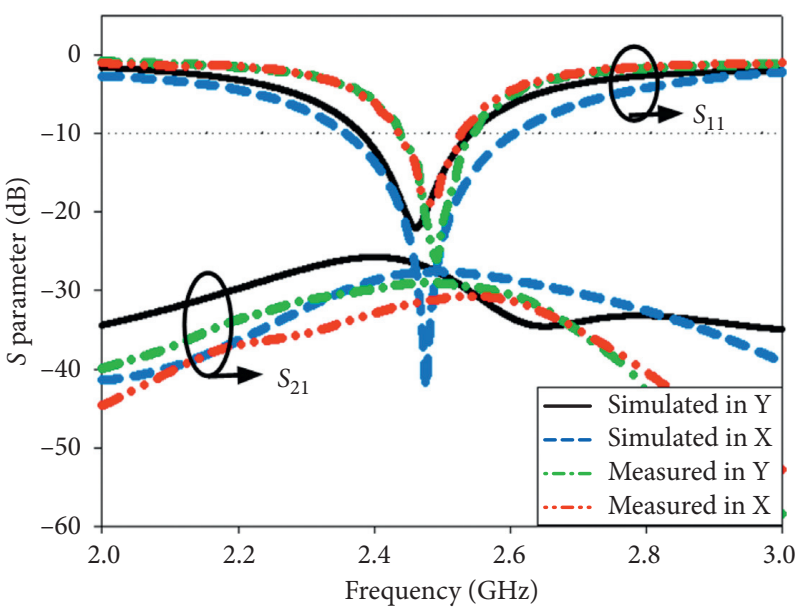

(b)

FIgURE 13: Simulated and measured result of $S_{11}$ and $S_{21}$ for circular bending: (a) $45 \mathrm{~mm}$ and (b) $55 \mathrm{~mm}$.

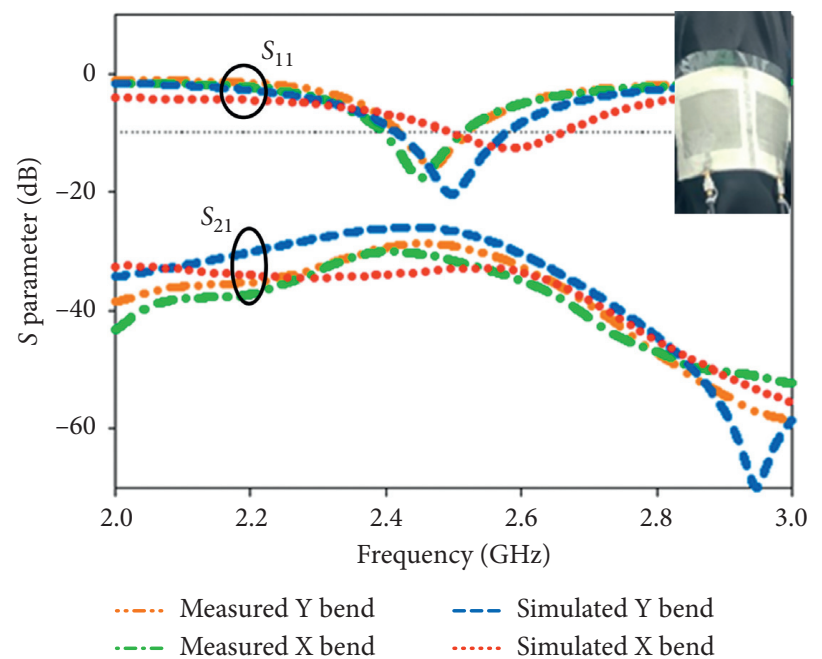

FIgURE 14: On-body measurement result of $S_{11}$ and $S_{21}$.

TABLE 4: Comparison of the experimental result of the wearable MIMO antenna.

\begin{tabular}{|c|c|c|c|c|c|c|c|c|}
\hline Ref & Element & Substrate & Freq $(\mathrm{GHz})$ & BW (\%) & $S_{21}(\mathrm{~dB})$ & Gain $(\mathrm{dBi})$ & MIMO gap & Complexity \\
\hline [5] & 3 & Polyamide $\left(\varepsilon_{r}=3.5\right)$ & 2.45 & 8 & 18 & -4.75 & $0.12 \lambda$ & Medium \\
\hline [13] & 2 & Jeans $\left(\varepsilon_{r}=1.6\right)$ & 3.1 & 129 & $>26$ & 6.9 & $0.052 \lambda$ & Medium \\
\hline [15] & 2 & Jeans $\left(\varepsilon_{r}=1.6\right)$ & 2.45 & 136 & $>22$ & 3.75 & $0.058 \lambda$ & Medium \\
\hline [14] & 2 & Felt $\left(\varepsilon_{r}=1.2\right)$ & 2.45 & 20 & $>15$ & 1.67 & Not mentioned & Medium \\
\hline Proposed & 2 & Felt $\left(\varepsilon_{r}=1.44\right)$ & 2.45 & 7 & $>26$ & 4.7 & $0.1 \lambda$ & Simple \\
\hline
\end{tabular}

[21]. In contrast with the proposed antenna in this paper, the body attachment experiment shifted the $S_{11}$ for about $250 \mathrm{MHz}$ to a lower frequency, as stated in [22]. It should be noted that with a simple and small MIMO gap, the proposed antenna performed well within the operating frequency. On top of that, this paper investigated the performance of the wearable MIMO antenna by folded bent, which has not been reported in previous works. The on-body measured results indicated that the antenna works well with close-proximity to the human body.

\section{Conclusions}

This work presented a simple two-element wearable MIMO antenna working in $2.45 \mathrm{GHz}$ for WBAN applications. The antenna performances were discussed in terms of $S$-parameter, radiation pattern, gain, ECC, and diversity gain. From the results, it could be seen that with $0.1 \lambda$ MIMO spacing, the antenna performed well in circular and folded bendings. Also, there were no significant changes in antenna performance in terms of $S_{11}$ for circular bending in $X$-axis, 
while better isolation was observed for the same axis bending. An isolation of at least $-26 \mathrm{~dB}$ was observed during circular bending in both $x$ - and $y$-axes. Conversely, in the case of folded bending, more variations in reflection coefficient $S_{11}$ result could be seen for $Y$-axis bend with an almost constant value of $S_{21}$. Furthermore, the proposed antenna exhibits a good diversity as the ECC is below 0.01 for all bending conditions.

\section{Data Availability}

No data were used to support this study.

\section{Conflicts of Interest}

The authors declare that they have no conflicts of interest.

\section{References}

[1] M. A. R. Osman, M. K. A. Rahim, N. A. Samsuri, M. K. Elbasheer, and M. E. Ali, "Textile UWB antenna bending and wet performances," International Journal of Antennas and Propagation, vol. 2012, Article ID 251682, 12 pages, 2012.

[2] M. El Gharbi, R. Fernández-García, S. Ahyoud, and I. Gil, “A review of flexible wearable antenna sensors: design, fabrication methods, and applications," Materials, vol. 13, no. 17, pp. 1-18, 2020.

[3] R. Joshi, E. F. N. M. Hussin, P. J. Soh et al., "Dual-band, dualsense textile antenna with AMC backing for localization using GPS and WBAN/WLAN," IEEE Access, vol. 8, pp. 8946889478, 2020.

[4] M. M. Ur Rashid, A. Rahman, L. C. Paul, and A. K. Sarkar, "Performance evaluation of a wearable $2.45 \mathrm{GHz}$ planar printed meandering monopole textile antenna on flexible substrates," in Proceedings of the 2019 1st International Conference on Advances in Science, Engineering and Robotics Technology, pp. 2-7, Dhaka, Bangladesh, May 2019.

[5] M. Alibakhshikenari, "A comprehensive survey of "metamaterial transmission-line based antennas: design, challenges, and applications," IEEE Access, vol. 8, pp. 144778-144808, 2020.

[6] M. Alibakhshikenari, "A comprehensive survey on "various decoupling mechanisms with focus on metamaterial and metasurface principles applicable to SAR and MIMO antenna systems," IEEE Access, vol. 8, pp. 192965-193004, 2020.

[7] M. Alibakhshikenari, "Antenna mutual coupling suppression over wideband using embedded periphery slot for antenna arrays," Electronics, vol. 7, no. 9, p. 198, 2018.

[8] M. Alibakhshikenari, "Compact single-layer traveling-wave antenna design using metamaterial transmission lines," Radio Science, vol. 52, no. 12, pp. 1510-1521, 2017.

[9] M. Alibakhshi-Kenari, "A new planar broadband antenna based on meandered line loops for portable wireless communication devices," Radio Science, vol. 51, no. 7, pp. 11091117, 2016.

[10] J. P. Xu, L. J. Xu, L. Ge, Z. Duan, and Y. M. Tang, "Flexible wearable antenna based on MIMO technology," in Proceedings of the 2019 IEEE MTT-S International Wireless Symposium, pp. 13-15, Guangzhou, China, May 2019.

[11] R. Sanchez-Montero, P. L. Lopez-Espi, C. Alen-Cordero, and J. A. Martinez-Rojas, "Bend and moisture effects on the performance of a U-shaped slotted wearable antenna for off- body communications in an industrial scientific medical (ISM) $2.4 \mathrm{GHz}$ band," Sensors, vol. 19, no. 8, 2019.

[12] J. Zhang, S. Yan, X. Hu, and G. A. E. Vandenbosch, "Reduction of mutual coupling for wearable antennas," in Proceedings of the 13th European Conference on Antennas and Propagation, EuCAP, Krakow, Poland, April 2019.

[13] H. L. Kao and C. H. Chuang, "Bending effects on a fabricbased antenna for wearable applications," in Proceedings of the 70th Electronic Components and Technology Conference, Orlando, FL, USA, June 2020.

[14] I. Adam, H. A. Rahim, M. N. M. Yasin, and M. N. M. Nasrol, "Mutual coupling suppression in wearable MIMO antenna for on/off-body WBAN applications," Journal of Physics: Conference Series, vol. 1755, no. 1, Article ID 12011, 2021.

[15] I. Adam, M. N. Mohd Yasin, N. Ramli et al., "Mutual coupling reduction of a wideband circularly polarized microstrip MIMO antenna," IEEE Access, vol. 7, pp. 97838-97845, 2019.

[16] E. Hadri, A. Z. Doae, A. Zugari, and M. El Ouahabi, "High isolation and ideal correlation using spatial diversity in a compact MIMO antenna for fifth-generation applications," International Journal of Antennas and Propagation, vol. 2020, Article ID 2740920, 10 pages, 2020.

[17] E. A. Mohammad, H. A. Rahim, P. J. Soh, M. F. Jamlos, M. Abdulmalek, and Y. S. Lee, "Dual-band circularly polarized textile antenna with split-ring slot for off-body $4 \mathrm{G} \mathrm{LTE}$ and WLAN applications," Applied Physics A, vol. 124, no. 8, 2018.

[18] H. A. Rahim, M. Abdulmalek, P. J. Soh, K. A. Rani, N. Hisham, and G. A. Vandenbosch, "Subject-specific effect of metallic body accessories on path loss of dynamic on-body propagation channels," Scientific Reports, vol. 6, Article ID 29818, 2016.

[19] D. Pifa, J. Wang, L. Zhao, G. Chen, Y. Wang, and W. Yu, "Ground plane effects on SAR for human head model exposed to a dual-band PIFA," in Proceedings of the IEEE MTT-S 2015 International Microwave Workshop Series on RF and Wireless Technologies for Biomedical and Healthcare Applications, Taipei, Taiwan, September 2015.

[20] A. Kumar Biswas and U. Chakraborty, "Compact wearable MIMO antenna with improved port isolation for ultrawideband applications," IET Microwaves, Antennas \& Propagation, vol. 13, no. 4, pp. 498-504, 2019.

[21] A. K. Biswas and U. Chakraborty, "A compact wide band textile MIMO antenna with very low mutual coupling for wearable applications," International Journal of $R F$ and Microwave Computer-Aided Engineering, vol. 29, no. 8, 2019.

[22] H. Li, S. Sun, B. Wang, and F. Wu, "Design of compact singlelayer textile MIMO antenna for wearable applications," IEEE Transactions on Antennas and Propagation, vol. 66, no. 6, pp. 3136-3141, 2018. 